\title{
Prevention in psychiatry
}

\section{Report of the Special Committee on the Place of Prevention in Psychiatry}

This report reviews the current status of prevention in psychiatry. A more detailed discussion of specific topics, derived from working papers prepared for the Committee, will in addition be published as an edited book by Gaskell Press.

In all areas of medicine, particularly within the NHS, emphasis is being directed more towards preventive approaches. The multifactorial causation of psychiatric disorders, and the remaining gaps in knowledge, mean that application is more complex. Balanced appraisal and evaluation are called for.

The general principles of prevention are first summarised in the report, together with some recent refinements. The classical framework of primary prevention to reduce incidence of new cases, secondary prevention to achieve early detection and treatment of presymptomatic cases, and tertiary prevention to lessen disability, is applicable to psychiatry. In psychiatry selective targeted approaches aimed at those most at risk appear more likely to be profitable than do universal approaches aimed at the whole population. Interventions aimed at the immediate social and family environment are more likely to succeed than those aimed at society as a whole. It is easier to modify precipitating rather than predisposing factors, and to enhance coping responses, rather than to eliminate risk factors. A wide range of biological and psychosocial factors contribute to causation of psychiatric disorders. Many have implications for prevention, ranging over genetic counselling, drug treatment to prevent recurrence, modification of adverse family environments, provision in adulthood of better social support, crisis intervention for major stressful life events, psychotherapeutic and cognitive approaches.

The report reviews preventive approaches to various specific disorders including depression, suicide, postnatal disorders, schizophrenia, anxiety disorders, eating disorders, drug and alcohol abuse. Prevention is reviewed in the psychiatric specialities of childhood disorders, mental handicap, old age psychiatry and forensic psychiatry, and in certain settings, such as general hospitals, general practice, the workplace, and developing countries.

The last chapter sets out general conclusions. There have been some striking successes in some areas, such as antenatal detection of specific genetic disorders causing mental handicap; use of maintenance medication to prevent recurrences of schizophrenia, depression and manic depressive disorder. A number of other areas are ready for targeted and evaluated studies. Selected examples include projects designed to ameliorate impact of major life events such as bereavement and life threatening catastrophes, using crisis intervention, counselling and cognitive-behavioural approaches; provision of supportive networks to those undergoing chronic adversity; studies of screening followed by appropriate intervention in general practice, at work, and in vulnerable groups such as postnatal mothers; detection and intervention for somatisers in general hospitals; impact of school-based health educational programmes regarding drugs, alcohol, sexual behaviour, severe dieting; community response programmes to limit drug use in affected communities; longer term studies using interventions in children of broken families, children in care, and children of psychiatrically ill parents. It is unlikely that preventive approaches currently available will lessen the need for treatment services, except in a few specific aspects such as severe mental handicap. The contribution of prevention is likely to grow progressively in the future as knowledge of causes increases. Evidence is not yet available to justify a large diversion of treatment resources to prevention and it is important that careful evaluation of pilot projects should precede any widespread introduction.

Chairman of the Special Committee on the Place of Prevention in Psychiatry

July 1993

The full Report is available from the College Publications Department (reference CR21, price £2) 